\title{
Successful Treatment of Rituximab-Resistant Epstein-Barr Virus-Associated Post-transplant Lymphoproliferative Disorder Using R-CHOP
}

\author{
Takuro Kuriyama, Noriaki Kawano, Kiyoshi Yamashita, and Akira Ueda
}

Epstein-Barr virus (EBV)-associated post-transplant lymphoproliferative disorder (EBV-PTLD) is a complication of hematopoietic stem cell transplantation (HSCT). Standard initial treatment of patients with EBV-PTLD includes administration of rituximab or dose reduction of a calcineurin inhibitor. We report successful chemotherapeutic treatment of rituximab-resistant EBV-PTLD after HSCT in a patient with severe aplastic anemia (AA). A 38-year-old woman with antithymocyte globulin (ATG)-resistant severe AA received bone marrow transplantation from an unrelated donor (human leukocyte antigen-DR singlelocus mismatch). The conditioning regimen included fludarabine, cyclophosphamide, ATG, and total body irradiation, and prophylaxis for graft-versus-host disease consisted of short methotrexate and tacrolimus. Neutrophil engraftment occurred on day 21. Left cervical lymph node swelling was observed after day 45, and analysis of a biopsy specimen revealed EBV-PTLD and a high blood EBV load (56,000 copies). The patient was treated with rituximab 4 times per week, but the lymphadenopathy continued and the blood EBV load increased to 96,000 copies. Half-dose treatment with rituximab, cyclophosphamide, vincristine, doxorubicin, and prednisolone (R-CHOP) was initiated on day 71. After 32 days of treatment with R-CHOP, the patient's neutrophil level was restored to $>0.5 \times 10^{9} / \mathrm{L}$ and both the lymphadenopathy and the blood EBV load ( $<100$ copies) were rapidly reduced. Although chemotherapy is not preferred soon after HSCT, it may be an effective strategy for treating patients with rituximab-resistant EBV-PTLD. [ $J$ Clin Exp Hematop 54(2) : 149-153, 2014]

Keywords: Epstein-Barr virus, hematopoietic stem cell transplantation, post-transplant lymphoproliferative disorder

\section{INTRODUCTION}

Hematopoietic stem cell transplantation (HSCT) is a recommended treatment option for patients with severe or very severe aplastic anemia (AA) who are 40 years of age or younger if they have a human leukocyte antigen (HLA)identical sibling donor. ${ }^{1}$ If there is no HLA-identical sibling donor, antithymocyte globulin (ATG) and cyclosporine are first-line treatments; however, recalcitrant cases must often undergo transplantation anyway. There are no clear guidelines for a conditioning regimen for patients with AA; however, in general, a conditioning regimen consisting of fludarabine, cyclophosphamide, ATG, or alemtuzumab and total body irradiation (TBI) is recommended. ${ }^{2,3}$ A conditioning

Received: May 16, 2014

Revised : June 10, 2014

Accepted: July 29, 2014

Department of Internal Medicine, Miyazaki Prefectural Miyazaki Hospital, Miyazaki, Japan

Corresponding author: Takuro Kuriyama

Department of Internal Medicine, Miyazaki Prefectural Miyazaki Hospital, 5-30 Kitatakamatsu-machi, Miyazaki 880-8510, Japan

E-mail: t-kuri@pref-hp.miyazaki.miyazaki.jp regimen that includes ATG improves graft rejection but increases the risk of complications such as viral infection, including reactivation of cytomegalovirus and Epstein-Barr virus $(\mathrm{EBV}){ }^{4}$

EBV-associated post-transplant lymphoproliferative disorder (EBV-PTLD) is a complication of HSCT. ${ }^{5,6}$ Immunosuppression, especially T-cell dysfunction, is important in the pathogenesis of EBV-PTLD, and EBV-PTLD usually occurs within 1 year after HSCT, before reconstitution of the EBV-specific cytotoxic T-lymphocyte response. ${ }^{7}$ The risk factors for EBV-PTLD are HLA mismatch between donor and recipient, conditioning regimens that include ATG, and T-cell-depleted transplantation. ${ }^{6-10}$ EBV DNA load is elevated exponentially in patients with EBV-PTLD, and weekly screening of the EBV DNA level is recommended for at least 3 months in high-risk patients who have undergone HSCT. ${ }^{11-13}$

Reducing immunosuppression is the usual first-line treatment for patients with EBV-PTLD. ${ }^{14}$ However, it is often difficult to reduce immunosuppression in the early stages after HSCT because of concerns about exacerbation of graftversus-host disease (GVHD) and graft rejection. Recently, rituximab, an anti-CD20 monoclonal antibody, has been used 
for the treatment of patients with EBV-PTLD, and response rates of $44-100 \%$ have been reported. ${ }^{15-19}$ However, these reports included patients treated with solid organ transplantation (SOT), and the effectiveness of treatment with rituximab limited to patients who have undergone HSCT remains unclear. Donor lymphocyte infusion (DLI) or chemotherapy is used for the treatment of rituximab-resistant EBV-PTLD, but there is no definitive therapy for this disease. Specifically, there is no established therapy for rituximab-resistant EBVPTLD after HSCT. We report the successful chemotherapeutic treatment of a patient with rituximab-resistant EBV-PTLD after HSCT using ATG for severe AA.

\section{CASE REPORT}

A 38-year-old Japanese woman was referred to our hospital because of severe AA; she gave informed consent to publish the details of her case. On admission, her blood cell counts had not yet recovered 1 year after treatment with ATG and cyclosporine. Results of laboratory studies included the following: hemoglobin, $6.1 \mathrm{~g} / \mathrm{dL}$; reticulocytes, $13.9 \times$ $10^{9} / \mathrm{L}$; platelets, $9.0 \times 10^{9} / \mathrm{L}$; white blood cell count, $0.98 \times$ $10^{9} / \mathrm{L}$ (neutrophils, $0.33 \times 10^{9} / \mathrm{L}$ ); blood urea nitrogen, 14.6 $\mathrm{mg} / \mathrm{dL}$; creatinine, $0.6 \mathrm{mg} / \mathrm{dL}$; total bilirubin, $0.63 \mathrm{mg} / \mathrm{dL}$; aspartate aminotransferase, $25 \mathrm{U} / \mathrm{L}$; alanine aminotransferase, $40 \mathrm{U} / \mathrm{L}$; lactase dehydrogenase, $139 \mathrm{U} / \mathrm{L}$; C-reactive protein, $0.09 \mathrm{mg} / \mathrm{dL}$; and ferritin, 1,002 ng/dL. She had a history of infection with EBV; EBV-viral capsid antigen immunoglobulin $\mathrm{M}$ antibody was negative, EBV-viral capsid antigen immunoglobulin $\mathrm{G}$ antibody was positive, and EBV DNA load was
$<100$ copies in whole blood. We obtained the patient's agreement for HSCT, which she received from an HLA-DR, single-locus, mismatched unrelated donor because she had no siblings or an HLA-matched unrelated donor. She received a conditioning regimen of $30 \mathrm{mg} / \mathrm{m}^{2}$ fludarabine (days -7 to -4), $750 \mathrm{mg} / \mathrm{m}^{2}$ cyclophosphamide (days -7 to -4 ), $2.5 \mathrm{mg}$ / $\mathrm{kg}$ ATG (days -3 and -2 ), and 2 Gy of TBI (day -1). Prophylaxis for GVHD consisted of tacrolimus and short methotrexate. The donor was male, his blood type was $\mathrm{AB}^{+}$ (recipient: $\mathrm{B}^{+}$major mismatch), and donor cell level was $1.70 \times 10^{8}$ cells $/ \mathrm{kg}$.

The clinical course is shown in Fig. 1. The patient developed a high fever when she received ATG on day -3 , but was afebrile the next day, and there were no other adverse events during conditioning chemotherapy. Tacrolimus was given from day -1 , and methotrexate was given on days $1,3,6$, and 11. There were no grade $\geq 3$ adverse events other than hematological toxicity and febrile neutropenia until neutrophil engraftment. Engraftment of neutrophils $\left(>0.5 \times 10^{9} / \mathrm{L}\right)$ was achieved on day 21. Chimerism analysis showed complete donor type on day 34 , and no grade $\geq 2$ GVHD occurred.

On day 43 , the patient developed a fever with swelling of her left tonsil. At first, we considered tonsillitis and she was treated with an oral antibiotic, but the swelling did not improve and left cervical lymphadenopathy appeared. Measurement of the EBV DNA level showed an increase to 56,000 copies (from $<100$ copies on day 21). A biopsy of the tonsil was performed; histopathological analysis showed that the normal structure was destroyed, with diffuse invasion of cells showing atypical nuclear bodies. With immunostain-

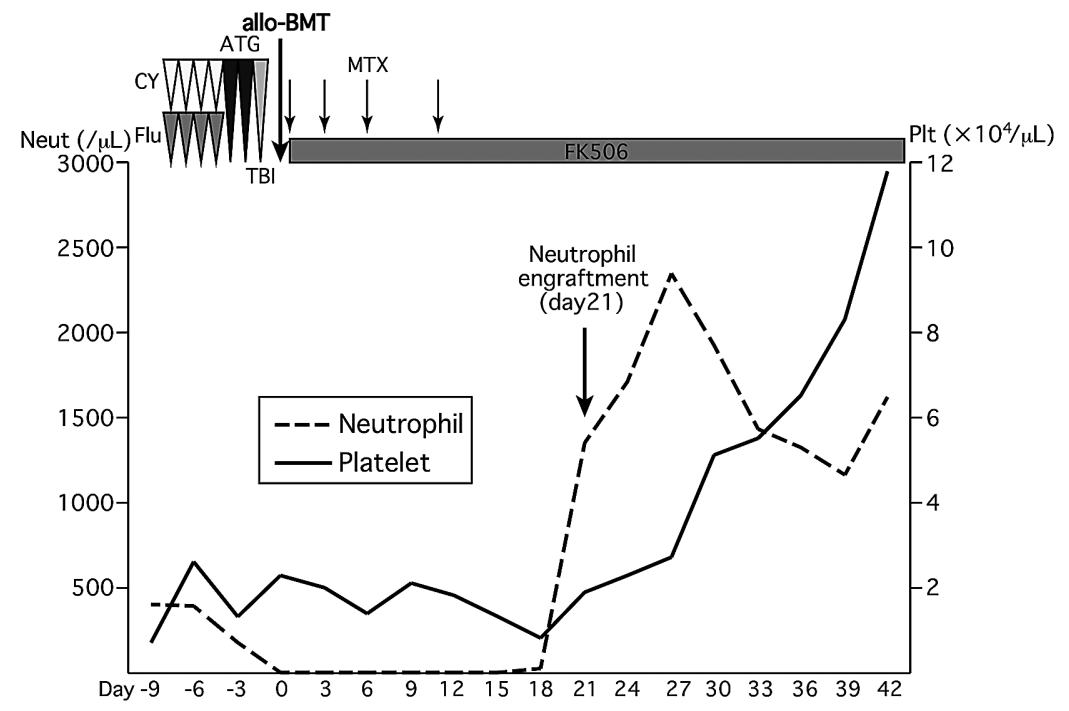

Fig. 1. The course of hematopoietic stem cell transplantation. Neut, neutrophil; Plt, platelets; CY, cyclophosphamide; Flu, fludarabine; ATG, antithymocyte globulin; TBI, total body irradiation; allo-BMT, allogeneic bone marrow transplantation; MTX, methotrexate; FK506, tacrolimus 
ing, CD10, CD20, CD30, and EBV-encoded small RNA were positive and the MIB-1 index was $70 \%$. EBV-PTLD was diagnosed on the basis of these results, and the patient was treated with rituximab $\left(375 \mathrm{mg} / \mathrm{m}^{2}\right)$ weekly from day 50 . Treatment with tacrolimus was slowly tapered without exacerbating the GVHD; however, the patient's tonsil swelling and cervical lymphadenopathy did not improve. After 3 administrations of rituximab, computed tomography (CT) revealed exacerbation of the tonsil swelling and cervical lymphadenopathy compared with the case before rituximab therapy, and the EBV DNA level had increased to 96,000 copies. The patient was treated with R-CHOP (rituximab: $375 \mathrm{mg} / \mathrm{m}^{2}$ on day 1; cyclophosphamide: $375 \mathrm{mg} / \mathrm{m}^{2}$ on day 2; vincristine: $0.7 \mathrm{mg} / \mathrm{m}^{2}$ on day 2; doxorubicin: $25 \mathrm{mg} / \mathrm{m}^{2}$ on day 2; and prednisolone: $50 \mathrm{mg}$ on days 2-6) at a $50 \%$ dose as second-line therapy for rituximab-resistant EBVPTLD from day 71. Although grade 4 neutropenia and thrombocytopenia were prolonged for more than 4 weeks, the patient's tonsil swelling and cervical lymphadenopathy improved and responded well to chemotherapy (Fig. 2). After recovery from the myelosuppression, $\mathrm{CT}$ and positron emission tomography/CT revealed resolution of the tonsil swelling and cervical lymphadenopathy, and a rapid reduction in the blood EBV load was observed ( $<100$ copies) (Fig. 3). Thereafter, the patient was treated with rituximab weekly for 4 doses and was discharged from our hospital on day 125 .

\section{DISCUSSION}

Standard therapy for EBV-PTLD after HSCT is rituximab or reduced immunosuppression therapy. However, it is difficult to reduce immunosuppression early after HSCT because of concerns about exacerbating GVHD; for this reason, rituximab is often chosen for the treatment of patients with EBVPTLD. The treatment of rituximab-resistant EBV-PTLD has not yet been established. With SOT, $63 \%$ of patients who developed localized EBV-PTLD improved with radiation therapy and $65-90 \%$ of patients with EBV-PTLD responded to standard CHOP or R-CHOP. ${ }^{20-22}$ In pediatric patients with rituximab-resistant EBV-PTLD after SOT, there was an $83 \%$ overall response rate to low-dose chemotherapy (cyclophosphamide $600 \mathrm{mg} / \mathrm{m}^{2}$ and prednisolone every 3 weeks for 6 cycles). ${ }^{23}$ There are some reports of the treatment of EBVPTLD after SOT or in pediatric patients, but there are only a few reports of treatment of EBV-PTLD after HSCT, especially during the early phase after HSCT. If chemotherapy is used for treatment, decisions regarding the therapeutic dose and frequency of treatment are currently made by the individual institution.

In our case, therapeutic options included DLI or chemotherapy when rituximab alone was ineffective for the treatment of EBV-PTLD. We chose chemotherapy because the patient's hematopoietic function had recovered (neutrophils $>$ $2.0 \times 10^{9} / \mathrm{L}$, platelets $>100 \times 10^{9} / \mathrm{L}$ ) and we were concerned that DLI might lead to unnecessary exacerbation of GVHD for AA. However, the optimal dosage of chemotherapy is unclear, and we chose a $50 \%$ dose reduction for R-CHOP. Despite the reduced dose, myelosuppression was greater than that with standard R-CHOP dosing for malignant lymphoma, and grade 4 neutropenia and thrombocytopenia were prolonged for 4 weeks. Although the single cycle of chemotherapy improved the cervical lymphadenopathy and blood EBV load, the number of cycles to use was unknown. We considered maintenance therapy with rituximab to be a reasonable

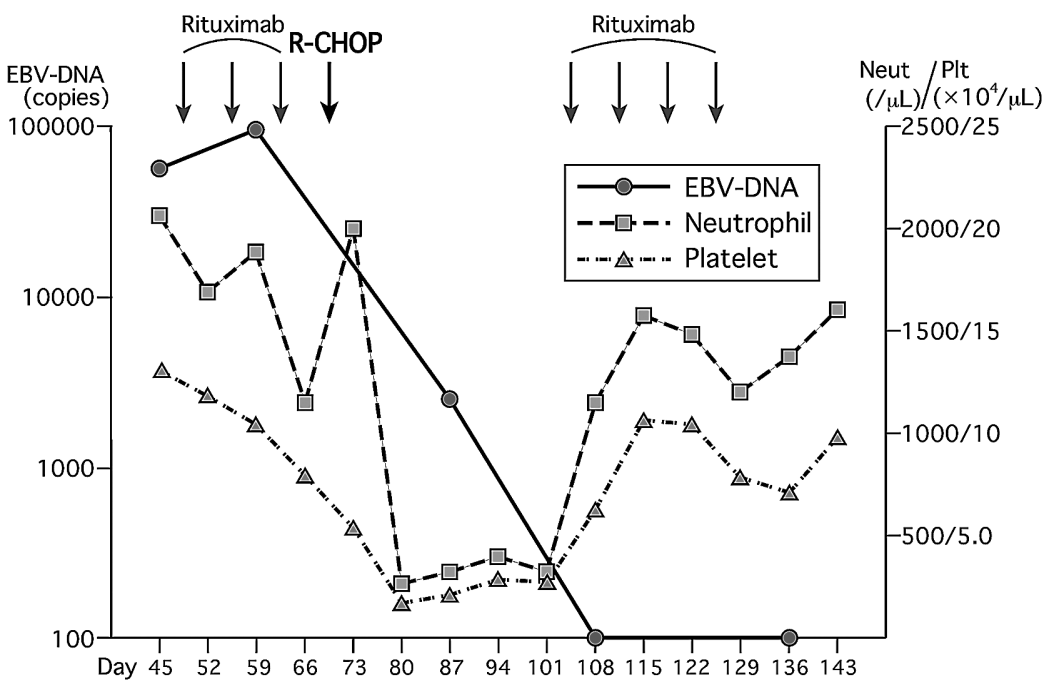

Fig. 2. The course of treatment of Epstein-Barr virus-associated post-transplant lymphoproliferative disorder. Neut, neutrophil; Plt, platelets; R-CHOP, rituximab, cyclophosphamide, vincristine, doxorubicin, prednisolone 

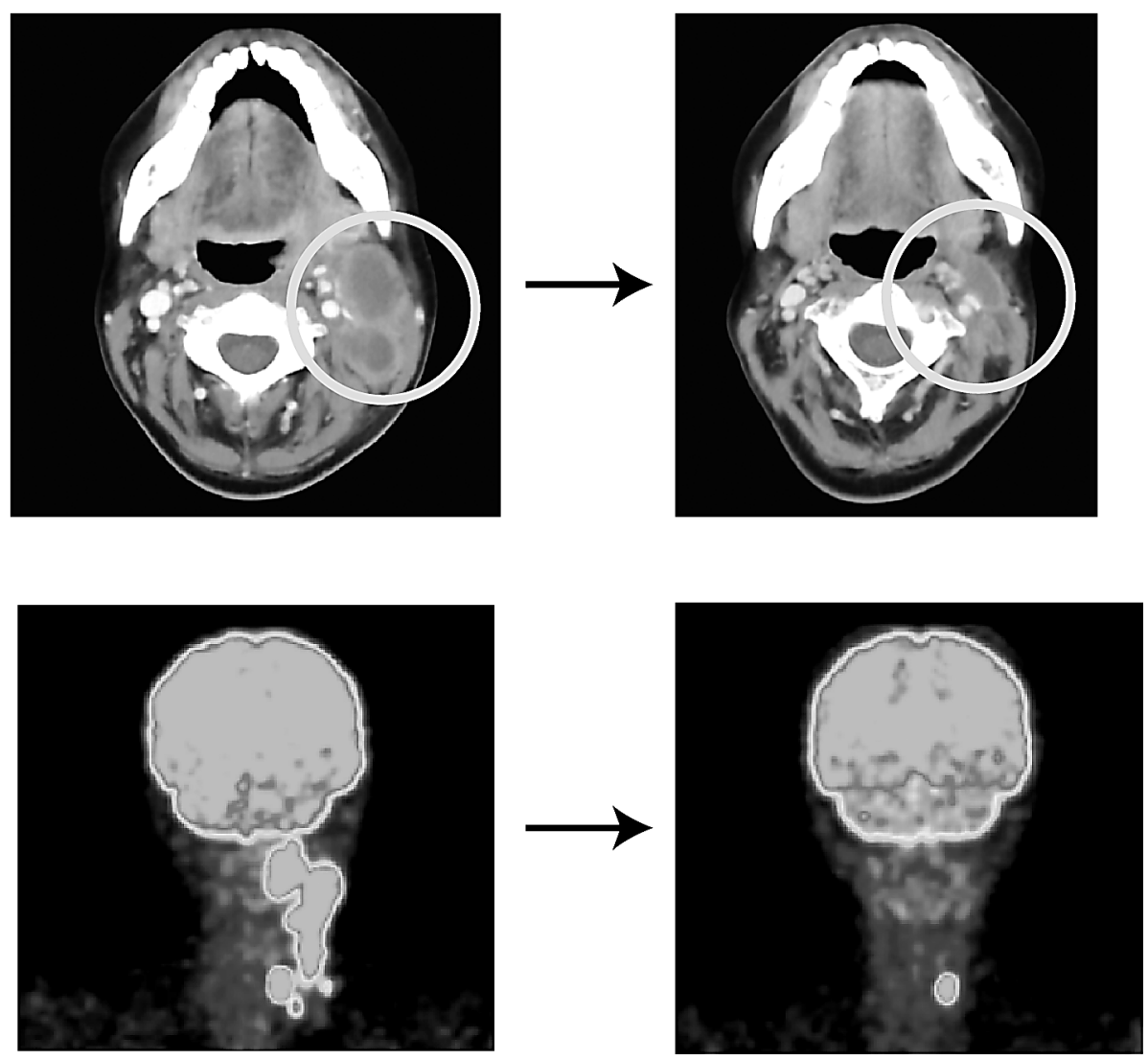

Fig. 3. Computed tomography and positron emission tomography images before and after treatment. Computed tomography and positron emission tomography revealed resolution of the tonsil swelling and cervical lymphadenopathy. There was a left thyroid mass before hematopoietic stem cell transplantation and cytological analysis revealed a benign disorder.

choice of therapy because the patient achieved remission with only a single course of R-CHOP, myelosuppression was greater than with standard R-CHOP dosing for malignant lymphoma and it would be difficult for the patient to receive multiple courses of chemotherapy, even though initial therapy with rituximab was ineffective for EBV-PTLD. After treatment, cervical lymphadenopathy did not recur in this patient and the blood EBV load has remained negative.

The response rate of DLI for EBV-PTLD is $>70 \%$, but it is associated with an increased risk of exacerbating GVHD because the frequency of alloreactive $\mathrm{T}$ cells exceeds that of EBV-specific T cells. ${ }^{24}$ Recent reports have shown that rituximab-resistant EBV-PTLD can be treated with EBVspecific T cells. ${ }^{25-28}$ Administration of EBV-specific T cells has few adverse effects, is effective, and prevents the development of EBV-PTLD. However, the cultivation of EBVspecific $\mathrm{T}$ cells takes more than 1 month and these cells cannot be reliably cultured and grown.

In conclusion, rituximab-resistant EBV-PTLD after HSCT for AA was treated with R-CHOP. Myelosuppression was prolonged for 4 weeks, although a single cycle of chemother- apy improved the EBV-PTLD. It is expected that diversification of the transplant source, including an HLAhaploidentical donor, is associated with increased potent immunosuppression, including the administration of ATG; as a result, there is the potential for an increase in the development of EBV-PTLD. With the reported efficacy of administering EBV-specific $T$ cells, a wide range of clinical applications is expected.

\section{CONFLICT OF INTEREST}

The authors declare no competing financial interests.

\section{REFERENCES}

1 Marsh JC, Ball SE, Cavenagh J, Darbyshire P, Dokal I, et al.: Guidelines for the diagnosis and management of aplastic anaemia. Br J Haematol 147:43-70, 2009

2 Gupta V, Ball SE, Sage D, Ortin M, Freires M, et al.: Marrow transplants from matched unrelated donors for aplastic anaemia using alemtuzumab, fludarabine and cyclophosphamide based 
conditioning. Bone Marrow Transplant 35:467-471, 2005

3 Bacigalupo A, Locatelli F, Lanino E, Marsh J, Socie G, et al.: Fludarabine, cyclophosphamide and anti-thymocyte globulin for alternative donor transplants in acquired severe aplastic anemia: a report from the EBMT-SAA Working Party. Bone Marrow Transplant 36:947-950, 2005

4 Scheinberg P, Fischer SH, Li L, Nunez O, Wu CO, et al:: Distinct EBV and CMV reactivation patterns following antibody-based immunosuppressive regimens in patients with severe aplastic anemia. Blood 109:3219-3224, 2007

5 Micallef IN, Chhanabhai M, Gascoyne RD, Shepherd JD, Fung $\mathrm{HC}$, et al.: Lymphoproliferative disorders following allogeneic bone marrow transplantation: the Vancouver experience. Bone Marrow Transplant 22:981-987, 1998

6 Shapiro RS, McClain K, Frizzera G, Gajl-Peczalska KJ, Kersey $\mathrm{JH}$, et al.: Epstein-Barr virus associated B cell lymphoproliferative disorders following bone marrow transplantation. Blood 71 : 1234-1243, 1988

7 Heslop HE: How I treat EBV lymphoproliferation. Blood 114: 4002-4008, 2009

8 Cohen JM, Cooper N, Chakrabarti S, Thomson K, Samarasinghe $\mathrm{S}$, et al:: EBV-related disease following haematopoietic stem cell transplantation with reduced intensity conditioning. Leuk Lymphoma 48:256-269, 2007

9 Brunstein CG, Weisdorf DJ, DeFor T, Barker JN, Tolar J, et al: Marked increased risk of Epstein-Barr virus-related complications with the addition of antithymocyte globulin to a nonmyeloablative conditioning prior to unrelated umbilical cord blood transplantation. Blood 108:2874-2880, 2006

10 van Esser JW, van der Holt B, Meijer E, Niesters HG, Trenschel $\mathrm{R}$, et al.: Epstein-Barr virus (EBV) reactivation is a frequent event after allogeneic stem cell transplantation (SCT) and quantitatively predicts EBV-lymphoproliferative disease following Tcell- -depleted SCT. Blood 98:972-978, 2001

11 Rooney CM, Loftin SK, Holladay MS, Brenner MK, Krance RA, et al.: Early identification of Epstein-Barr virus-associated posttransplantation lymphoproliferative disease. Br J Haematol 89:98103, 1995

12 Styczynski J, Reusser P, Einsele H, de la Camara R, Cordonnier C, et al:: Management of HSV, VZV and EBV infections in patients with hematological malignancies and after SCT: guidelines from the Second European Conference on Infections in Leukemia. Bone Marrow Transplant 43:757-770, 2009

13 Kimura H, Ito Y, Suzuki R, Nishiyama Y: Measuring EpsteinBarr virus (EBV) load: the significance and application for each EBV-associated disease. Rev Med Virol 18:305-319, 2008

14 Heslop HE, Li C, Krance RA, Loftin SK, Rooney CM: EpsteinBarr infection after bone marrow transplantation. Blood 83:17061708, 1994

15 Faye A, Van Den Abeele T, Peuchmaur M, Mathieu-Boue A, Vilmer E: Anti-CD20 monoclonal antibody for post-transplant lymphoproliferative disorders. Lancet 352:1285, 1998
16 Verschuuren EA, Stevens SJ, van Imhoff GW, Middeldorp JM, de Boer C, et al:: Treatment of posttransplant lymphoproliferative disease with rituximab: the remission, the relapse, and the complication. Transplantation 73:100-104, 2002

17 Ganne V, Siddiqi N, Kamaplath B, Chang CC, Cohen EP, et al.: Humanized anti-CD20 monoclonal antibody (Rituximab) treatment for post-transplant lymphoproliferative disorder. Clin Transplant 17:417-422, 2003

18 Blaes AH, Peterson BA, Bartlett N, Dunn DL, Morrison VA: Rituximab therapy is effective for posttransplant lymphoproliferative disorders after solid organ transplantation: results of a phase II trial. Cancer 104:1661-1667, 2005

19 Oertel SH1, Verschuuren E, Reinke P, Zeidler K, Papp-Váry M, et $a l$ : : Effect of anti-CD20 antibody rituximab in patients with posttransplant lymphoproliferative disorder (PTLD). Am J Transplant 5:2901-2906, 2005

20 Dotti G, Fiocchi R, Motta $\mathrm{T}$, Mammana C, Gotti E, et al.: Lymphomas occurring late after solid-organ transplantation: influence of treatment on the clinical outcome. Transplantation 74: 1095-1102, 2002

21 Choquet S, Trappe R, Leblond V, Jäger U, Davi F, et al.: CHOP21 for the treatment of post-transplant lymphoproliferative disorders (PTLD) following solid organ transplantation. Haematologica 92:273-274, 2007

22 Trappe R, Oertel S, Leblond V, Mollee P, Sender M, et al.: Sequential treatment with rituximab followed by CHOP chemotherapy in adult B-cell post-transplant lymphoproliferative disorder (PTLD): the prospective international multicentre phase 2 PTLD-1 trial. Lancet Oncol 13:196-206, 2012

23 Gross TG, Bucuvalas JC, Park JR, Greiner TC, Hinrich SH, et al.: Low-dose chemotherapy for Epstein-Barr virus-positive posttransplantation lymphoproliferative disease in children after solid organ transplantation. J Clin Oncol 23:6481-6488, 2005

24 Heslop HE, Brenner MK, Rooney CM: Donor T cells to treat EBV-associated lymphoma. N Engl J Med 331:679-680, 1994

25 Rooney CM, Smith CA, Ng CY, Loftin SK, Sixbey JW, et al.: Infusion of cytotoxic $\mathrm{T}$ cells for the prevention and treatment of Epstein-Barr virus-induced lymphoma in allogeneic transplant recipients. Blood 92:1549-1555, 1998

26 Heslop HE, Ng CY, Li C, Smith CA, Loftin SK, et al:: Longterm restoration of immunity against Epstein-Barr virus infection by adoptive transfer of gene-modified virus-specific $\mathrm{T}$ lymphocytes. Nat Med 2:551-555, 1996

27 Comoli P, Basso S, Zecca M, Pagliara D, Baldanti F, et al.: Preemptive therapy of EBV-related lymphoproliferative disease after pediatric haploidentical stem cell transplantation. Am J Transplant 7:1648-1655, 2007

28 O'Reilly RJ, Doubrovina E, Trivedi D, Hasan A, Kollen W, et al.: Adoptive transfer of antigen-specific T-cells of donor type for immunotherapy of viral infections following allogeneic hematopoietic cell transplants. Immunol Res 38:237-250, 2007 